\title{
Design of Ultraspherical Window Functions with Prescribed Spectral Characteristics
}

\author{
Stuart W. A. Bergen \\ Department of Electrical and Computer Engineering, University of Victoria, P.O. Box 3055 STN CSC, \\ Victoria, BC, Canada V8W $3 P 6$ \\ Email: sbergen@ece.uvic.ca \\ Andreas Antoniou \\ Department of Electrical and Computer Engineering, University of Victoria, P.O. Box 3055 STN CSC, \\ Victoria, BC, Canada V8W 3P6 \\ Email: aantoniou@ieee.org
}

Received 7 April 2003; Revised 17 January 2004; Recommended for Publication by Hideaki Sakai

\begin{abstract}
A method for the design of ultraspherical window functions that achieves prescribed spectral characteristics is proposed. The method comprises a collection of techniques that can be used to determine the three independent parameters of the ultraspherical window such that a specified ripple ratio and main-lobe width or null-to-null width along with a user-defined side-lobe pattern can be achieved. Other known two-parameter windows can achieve a specified ripple ratio and main-lobe width; however, their side-lobe pattern cannot be controlled as in the proposed method. A comparison with other windows has shown that a difference in performance exists between the ultraspherical and Kaiser windows, which depends critically on the required specifications. The paper also highlights some applications of the proposed method in the areas of digital beamforming and image processing.
\end{abstract}

Keywords and phrases: window functions, ultraspherical window, beamforming, image processing, digital filters.

\section{INTRODUCTION}

Windows are time-domain weighting functions that are used to reduce Gibbs' oscillations resulting from the truncation of a Fourier series. Their roots date back over one-hundred years to Fejer's averaging technique for a truncated Fourier series and they are employed in a variety of traditional signal processing applications including power spectral estimation, beamforming, and digital filter design. Despite their maturity, windows functions (or windows for short) continue to find new roles in the applications of today. Very recently, windows have been used to facilitate the detection of irregular and abnormal heartbeat patterns in patients in electrocardiograms $[1,2]$. Medical imaging systems, such as the ultrasound, have also shown enhanced performance when windows are used to improve the contrast resolution of the system [3]. Windows have also been employed to aid in the classification of cosmic data $[4,5]$ and to improve the reliability of weather prediction models [6]. With such a large number of applications available for windows that span a variety of disciplines, general methods that can be used to design windows with arbitrary characteristics are especially useful.

Windows can be categorized as fixed or adjustable [7]. Fixed windows have only one independent parameter, namely, the window length which controls the main-lobe width. Adjustable windows have two or more independent parameters, namely, the window length, as in fixed windows, and one or more additional parameters that can control other window characteristics $[8,9,10,11,12,13]$. The Kaiser and Saramäki windows $[8,9]$ have two parameters and achieve close approximations to discrete prolate functions that have maximum energy concentration in the main lobe. The Dolph-Chebyshev window [10] has two parameters and produces the minimum main-lobe width for a specified maximum side-lobe level. The Kaiser, Saramäki, and Dolph-Chebyshev windows can control the amplitude of the side lobes relative to that of the main lobe. The ultraspherical window has three parameters, and through the proper choice of these parameters, the amplitude of the side lobes relative to that of the main lobe can be controlled as in the Kaiser, Saramäki, and Dolph-Chebyshev windows; and in addition, arbitrary side-lobe patterns can be achieved. To facilitate the application of the ultraspherical window to the diverse range of applications alluded to earlier, a practical and efficient design method is required that can utilize its inherent flexibility.

In this paper, a method is proposed for designing ultraspherical windows that achieves prescribed spectral characteristics such as specified ripple ratio, main-lobe width, 
null-to-null width, and a user-defined side-lobe pattern. The paper is structured as follows. Section 2 presents some performance measures for windows. Section 3 introduces the ultraspherical window and some formulas for generating its coefficients from three independent parameters. Aspects of the window's frequency spectrum and its equivalence to other windows are also discussed. Section 4 proposes a method for designing ultraspherical windows that achieve prescribed spectral characteristics. The method entails a variety of short algorithms that calculate two of the three independent parameters based on the prescribed spectral characteristics. Section 5 proposes an empirical equation that can be used to accurately predict the window length required to achieve multiple prescribed spectral characteristics simultaneously. Section 6 compares the ultraspherical window's effectiveness in achieving prescribed spectral characteristics with respect to other conventional windows. Section 7 presents examples and demonstrates the accuracy of the proposed method. Section 8 describes two applications of the proposed method in the areas of beamforming and image processing. Section 9 provides concluding remarks.

\section{CHARACTERIZATION OF WINDOWS}

Windows are frequently compared and classified in terms of their spectral characteristics. The frequency spectrum of a window is given by

$$
W\left(e^{j \omega T}\right)=e^{-j \omega(N-1) T / 2} W_{0}\left(e^{j \omega T}\right),
$$

where $W_{0}\left(e^{j \omega T}\right)$ is called the amplitude function, $N$ is the window length, and $T$ is the interval between samples. The amplitude and phase spectrums of a window are given by $A(\omega)=\left|W_{0}\left(e^{j \omega T}\right)\right|$ and $\theta(\omega)=-\omega(N-1) T / 2$, respectively, and $\left|W_{0}\left(e^{j \omega T}\right)\right| / W_{0}\left(e^{0}\right)$ is a normalized version of the amplitude spectrum. The normalized amplitude spectrum of a typical window is depicted in Figure 1.

Two parameters of windows in general are the null-tonull width $B_{n}$ and the main-lobe width $B_{r}$. These quantities are defined as $B_{n}=2 \omega_{n}$ and $B_{r}=2 \omega_{r}$, where $\omega_{n}$ and $\omega_{r}$ are the half null-to-null and half main-lobe widths, respectively, as shown in Figure 1. An important window parameter is the ripple ratio $r$ which is defined as

$$
r=\frac{\text { maximum side-lobe amplitude }}{\text { main-lobe amplitude }}
$$

(see Figure 1). The ripple ratio is a small quantity less than unity and, in consequence, it is convenient to work with the reciprocal of $r$ in $\mathrm{dB}$, that is,

$$
R=20 \log \left(\frac{1}{r}\right)
$$

$R$ can be interpreted as the minimum side-lobe attenuation relative to the main lobe and $-R$ is the ripple ratio in $d B$. Another parameter that may be used to quantify a window's side-lobe pattern is the side-lobe roll-off ratio, $s$, which is defined as

$$
s=\frac{a_{1}}{a_{2}}
$$

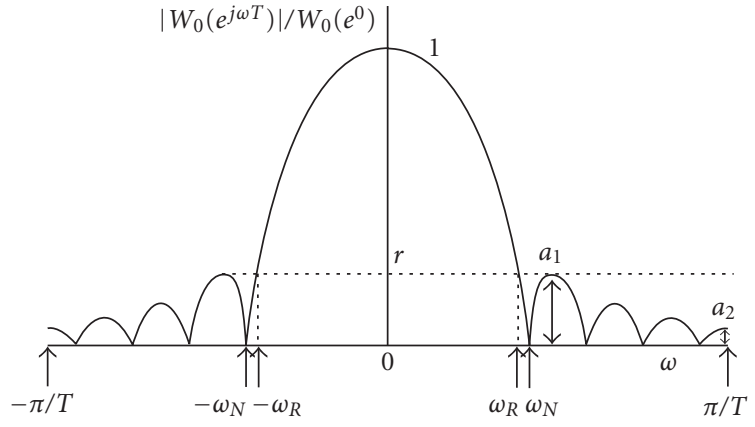

FIGURE 1: A typical window's normalized amplitude spectrum and some common spectral characteristics.

where $a_{1}$ and $a_{2}$ are the amplitudes of the side lobe nearest and furthest, respectively, from the main lobe (see Figure 1). If $S$ is the side-lobe roll-off ratio in $\mathrm{dB}$, then $s$ is given by

$$
s=10^{S / 20} .
$$

For the side-lobe roll-off ratio to have meaning, the envelope of the side-lobe pattern should be monotonically increasing or decreasing.

These spectral characteristics are important performance measures for windows. When analyzing narrowband signals, such as sinusoids, weak signals can easily be obscured by nearby strong signals. The width characteristics provide a resolution measure between adjacent signals while the ripple ratio determines the worst-case scenario for detecting weak signals in the presence of strong narrowband signals. The side-lobe roll-off ratio provides a simple description of the distribution of energy throughout the side lobes, which can be of importance if prior knowledge of the location of an interfering signal is known. Further explanation of the usefulness of these spectral characteristics can be found in [11].

\section{THE ULTRASPHERICAL WINDOW}

The coefficients of a right-sided ultraspherical window of length $N$ can be calculated explicitly as $[12,14]$

$$
\begin{aligned}
w(n T)= & \frac{A}{p-n}\left(\begin{array}{c}
\mu+p-n-1 \\
p-n-1
\end{array}\right) \\
& \cdot \sum_{m=0}^{n}\left(\begin{array}{c}
\mu+n-1 \\
n-m
\end{array}\right)\left(\begin{array}{c}
p-n \\
m
\end{array}\right) B^{m} \text { for } n=0,1, \ldots, N-1,
\end{aligned}
$$

where

$$
\begin{aligned}
A & = \begin{cases}\mu x_{\mu}^{p} & \text { for } \mu \neq 0, \\
x_{\mu}^{p} & \text { for } \mu=0,\end{cases} \\
B & =1-x_{\mu}^{-2}, \\
p & =N-1 .
\end{aligned}
$$

In (6) $\mu, x_{\mu}$, and $N$ are independent parameters and $w[(N-$ $n-1) T]=w(n T)$. A normalized window is obtained as 
$\hat{w}(n T)=w(n T) / w(C T)$ where

$$
C= \begin{cases}\frac{N-1}{2} & \text { for odd } N \\ \frac{N}{2-1} & \text { for even } N\end{cases}
$$

The binomial coefficients can be calculated as

$$
\left(\begin{array}{l}
\alpha \\
0
\end{array}\right)=1, \quad\left(\begin{array}{l}
\alpha \\
p
\end{array}\right)=\frac{\alpha(\alpha-1) \cdots(\alpha-p+1)}{p !} \quad \text { for } p \geq 1 .
$$

The independent parameter $x_{\mu}$ can be expressed as

$$
x_{\mu}=\frac{x_{N-1,1}^{(\mu)}}{\cos (\beta \pi / N)}
$$

where $\beta \geq 1$ and $x_{N-1,1}^{(\mu)}$ is the largest zero of the ultraspherical polynomial $C_{N-1}^{\mu}(x)$. The new independent parameter $\beta$ is the so-called shape parameter and can be used to set the null-to-null width of a window to $4 \beta \pi / N$, that is, $\beta$ times that of the rectangular window [9]. Throughout the paper, $x_{n, l}^{(\lambda)}$ denotes the $l$ th zero of the ultraspherical polynomial $C_{n}^{\lambda}(x)$. Unfortunately, closed-form expressions for the zeros of this polynomial do not exist but the zeros can be found quickly using the following iterative algorithm which is valid for $l=1$ and $\operatorname{rnd}(n / 2)$ yielding the largest and smallest zeros, respectively. The rounding operator is defined as

$$
\operatorname{rnd}(x)=\operatorname{int}(x+0.5)
$$

where $\operatorname{int}(y)$ is the integer part of $y$ and is also known as the floor operator. Due to the symmetry relation $C_{n}^{\mu}(-x)=$ $(-1)^{n} C_{n}^{\mu}(x)$, only the positive zeros need to be considered.

Algorithm 1 (lth zero of $\left.C_{n}^{\lambda}(x)\right)$.

Step 1

Input $l, \lambda, n$, and $\varepsilon$.

If $\lambda=0$, then output $x^{*}=\cos [\pi(l-1 / 2) / n]$ and stop. If $\lambda=1$, then output $x^{*}=\cos [l \pi /(n+1)]$ and stop. Set $k=1$, and compute

$$
y_{1}=\frac{\sqrt{n^{2}+2(n-1) \lambda-1}}{n+\lambda} \cos \frac{(l-1) \pi}{n-1} .
$$

Step 2

Compute

$$
y_{k+1}=y_{k}-\frac{C_{n}^{\lambda}\left(y_{k}\right)}{2 \lambda C_{n-1}^{\lambda+1}\left(y_{k}\right)} .
$$

The values of $C_{n}^{\lambda}(x)$ can be calculated using the recurrence relationship [15]

$$
\begin{aligned}
C_{r}^{\lambda}(x)=\frac{1}{r} & {\left[2 x(r+\lambda-1) C_{r-1}^{\lambda}(x)\right.} \\
& \left.-(r+2 \lambda-2) C_{r-2}^{\lambda}(x)\right]
\end{aligned}
$$

for $r=2,3, \ldots, n$, where $C_{0}^{\lambda}(x)=1$ and $C_{1}^{\lambda}(x)=2 \lambda x$. The denominator in (13) can be calculated quickly using the recurrence relationship [15]

$2 \lambda C_{r-1}^{\lambda+1}(x)=\frac{2 \lambda+r-1}{1-x^{2}} C_{r-1}^{\lambda}(x)-(r x) C_{r}^{\lambda}(x)$

which uses some of the intermediate calculations from (14).

Step 3

If $\left|y_{k+1}-y_{k}\right| \leq \varepsilon$, then output $x^{*}=y_{k+1}$ and stop.

Set $k=k+1$, and repeat from Step 2 .

In this algorithm, $\varepsilon$ is the termination tolerance. A good choice is $\varepsilon=10^{-6}$ which would cause the algorithm to converge in 3 to 6 iterations. Equation (12) in Step 1 represents the lowest upper bound for the zeros of the ultraspherical polynomial [16]. In Step 2, the Newton-Raphson method is used to obtain the next estimate of the zero.

The amplitude function of the ultraspherical window is given by

$$
W_{0}\left(e^{j \omega T}\right)=C_{N-1}^{\mu}\left[x_{\mu} \cos \left(\frac{\omega T}{2}\right)\right]
$$

where $C_{n}^{\mu}(x)$ is the ultraspherical polynomial which can be calculated using the recurrence relationship given in (14).

The Dolph-Chebyshev window is a special case of the ultraspherical window and can be obtained by letting $\mu=0$ in (6), which results in

$$
W_{0}\left(e^{j \omega T}\right)=T_{N-1}\left[x_{\mu} \cos \left(\frac{\omega T}{2}\right)\right]
$$

where

$$
T_{n}(x)=\cos \left(n \cos ^{-1} x\right)
$$

is the Chebyshev polynomial of the first kind. In the DolphChebyshev window, the side-lobe pattern is fixed, that is, (1) all side lobes have the same amplitude and (2) a minimum main-lobe width is achieved for a specified side-lobe level. Hence this window is usually designed to yield a specified ripple ratio $r$. To design a Dolph-Chebyshev window, $x_{\mu}$ is calculated using the relation [10]

$$
x_{\mu}=x_{0}=\cosh \left(\frac{1}{N-1} \cosh ^{-1} \frac{1}{r}\right) \text {. }
$$

Alternatively, the Dolph-Chebyshev window can be designed to yield a specified null-to-null width $\beta$ times that of the rectangular window. This can be accomplished by using (10) where $x_{N-1,1}^{(\mu)}=x_{N-1,1}^{(0)}$ is the largest zero of the Chebyshev polynomial of the first kind $T_{N-1}(x)$, which is given by

$$
x_{N-1,1}^{(0)}=\cos \left[\frac{\pi}{2(N-1)}\right] \text {. }
$$


The Saramäki window is a special case of the ultraspherical window and can be obtained by letting $\mu=1$ in (6), which results in

$$
W_{0}\left(e^{j \omega T}\right)=U_{N-1}\left[x_{\mu} \cos \left(\frac{\omega T}{2}\right)\right]
$$

where

$$
U_{n}(x)=\frac{\sin \left[(n+1) \cos ^{-1} x\right]}{\sin \left(\cos ^{-1} x\right)}
$$

is the Chebyshev polynomial of the second kind. The Saramäki window, like the Kaiser window, is known for achieving close approximations to discrete prolate functions and is designed to yield a null-to-null width $\beta$ times that of the rectangular window. This can be accomplished by using (10) where $x_{N-1,1}^{(\mu)}=x_{N-1,1}^{(1)}$ is the largest zero of the Chebyshev polynomial of the second kind $U_{N-1}(x)$, which is given by

$$
x_{N-1,1}^{(1)}=\cos \left(\frac{\pi}{N}\right) \text {. }
$$

Another special case of interest is the case where $\mu=1 / 2$ in (6), which results in

$$
W_{0}\left(e^{j \omega T}\right)=P_{N-1}\left[x_{\mu} \cos \left(\frac{\omega T}{2}\right)\right],
$$

where $P_{n}(x)$ is the Legendre polynomial which can be calculated using the recurrence relationship

$$
P_{r}(x)=\frac{1}{r}\left[x(2 r-1) P_{r-1}(x)-(r-1) P_{r-2}(x)\right]
$$

for $r=2,3, \ldots, n$, where $P_{0}(x)=1$ and $P_{1}(x)=x$.

\section{PRESCRIBED SPECTRAL CHARACTERISTICS}

With the appropriate selection of the parameters $\mu, x_{\mu}$, and $N$, ultraspherical windows can be designed to achieve prescribed specifications for the side-lobe roll-off ratio, the ripple ratio, and one of the two width characteristics simultaneously. Parameter $\mu$ alters the side-lobe roll-off ratio, $x_{\mu}$ provides a trade-off between the ripple ratio and a width characteristic, and $N$ allows different ripple ratios to be obtained for a fixed width characteristic and vice versa. In some applications the window length $N$ may be fixed. Such a scenario limits the designer's choice in achieving prescribed specifications for the side-lobe roll-off ratio and either the ripple ratio or a width characteristic but not both. For the case where $N$ is adjustable, a prediction of $N$ is possible which allows one to achieve prescribed specifications for the side-lobe roll-off ratio, the ripple ratio, and a width characteristic simultaneously.

In the subsections to follow, algorithms are proposed that achieve each prescribed specification to a high degree of precision. Some important quantities to be used are identified in Figure 2 which depicts a plot of $C_{N-1}^{\mu}(x)$ for the values $\mu=2$ and $N=7$. The modified sign (msgn) and max functions are

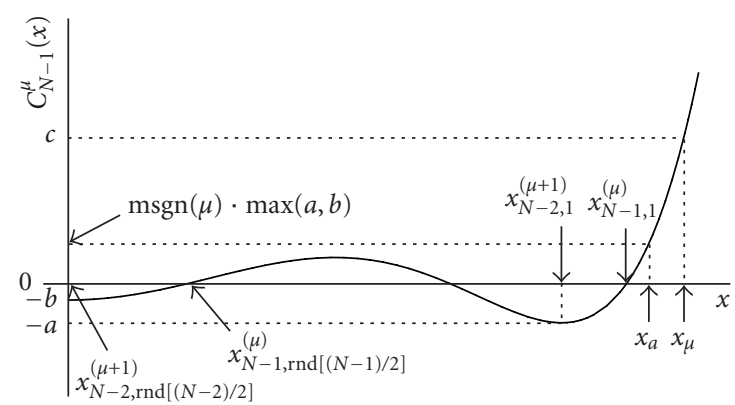

FIGURE 2: Some important quantities of the ultraspherical polynomial $C_{N-1}^{\mu}(x)$ for the values $\mu=2$ and $N=7$.

defined as

$$
\begin{gathered}
\operatorname{msgn}(x)= \begin{cases}-1 & \text { for } x<0 \\
1 & \text { for } x \geq 0\end{cases} \\
\max (x, y)= \begin{cases}x & \text { for } x \geq y \\
y & \text { for } y>x\end{cases}
\end{gathered}
$$

\subsection{Side-lobe roll-off ratio}

To generate an ultraspherical window for a fixed $N$ and a prescribed side-lobe roll-off ratio $s$, one can select the parameter $\mu$ appropriately. This can be accomplished by solving the one-dimensional minimization problem

$$
\operatorname{minimize}_{\mu_{L} \leq \mu \leq \mu_{H}} F=\left[s-\left|\frac{C_{N-1}^{\mu}\left(x_{N-2,1}^{(\mu+1)}\right)}{C_{N-1}^{\mu}\left(x_{N-2, \operatorname{rnd}[(N-2) / 2]}^{(\mu+1)}\right)}\right|\right]^{2},
$$

where the values of $C_{n}^{\mu}(x)$ are given by (14), and $x_{N-2,1}^{(\mu+1)}$ and $x_{N-2, \operatorname{rnd}[(N-2) / 2]}^{(\mu+1}$, which are identified in Figure 2, are the largest and smallest zeros, respectively, of the derivative of $C_{N-1}^{\mu}(x)$, namely, $2 \mu C_{N-2}^{\mu+1}(x)$. The zero $x_{N-2,1}^{(\mu+1)}$ can be found using Algorithm 1 with $l=1, \lambda=\mu+1, n=N-2$, and $\varepsilon=10^{-6}$. The zero $x_{N-2, \operatorname{mnd}[(N-2) / 2]}^{(\mu+1)}$ can be found using Algorithm 1 with $l=\operatorname{rnd}[(N-2) / 2], \lambda=\mu+1, n=N-2$, and $\varepsilon=10^{-6}$.

Simple algorithms such as dichotomous, Fibonacci, or golden section line searches, as outlined in [17], can be used to perform the minimization in (27). The lower and upper bounds on $\mu$ in (27) can be set to

$$
\begin{gathered}
\mu_{L}=0, \quad \mu_{H}=10, \quad \text { for } s>1, \\
\mu_{L}=-0.9999, \quad \mu_{H}=0, \quad \text { for } 0<s<1 .
\end{gathered}
$$

If $s=1$, then no minimization is necessary and $\mu=0$ yields the Dolph-Chebyshev window. The bound $\mu_{L}=-0.9999$ was chosen because $C_{N-1}^{\mu}(x)$ has a singularity at $\mu=-1$. Also, for values of $\mu \leq-1.5$, the zeros of the ultraspherical polynomial overlap rendering the resulting window useless for our purposes. The bound $\mu_{H}=10$ was chosen because the improvements in the side-lobe roll-off ratio that can be achieved for values of $\mu>10$ are negligible. 
TABLE 1: Limiting side-lobe roll-off ratios for small values of $N$.

\begin{tabular}{c|cc}
\hline$N$ & $\min S(\mathrm{~dB})$ & $\max S(\mathrm{~dB})$ \\
\hline 5 & -6.02 & 4.95 \\
6 & -7.65 & 7.88 \\
7 & -10.19 & 12.78 \\
8 & -11.43 & 16.25 \\
9 & -13.05 & 20.82 \\
10 & -14.02 & 24.32 \\
11 & -15.20 & 28.55 \\
12 & -16.00 & 31.93 \\
13 & -16.93 & 35.83 \\
14 & -17.61 & 39.05 \\
15 & -18.37 & 42.67 \\
16 & -18.96 & 45.72 \\
17 & -19.61 & 49.07 \\
18 & -20.13 & 51.96 \\
19 & -20.69 & 55.08 \\
20 & -21.15 & 57.81 \\
\hline
\end{tabular}

The ultraspherical window imposes limits on the sidelobe roll-off ratio that can be achieved for low values of $N$. For example, if $N=7$, window designs with $S=20 \log _{10} s$ outside the range $-10.19<S<12.78 \mathrm{~dB}$ are not possible for any value of $\mu$. For this reason, the side-lobe roll-off ratio's design range must be limited for a given $N$ to that produced using $\mu_{L}=-0.9999$ and $\mu_{H}=10$. The limiting values are shown in Table 1 for window lengths in the range $5 \leq N \leq 20$ which spans the practical design range $-20 \leq S \leq 60 \mathrm{~dB}$.

\subsection{Null-to-null width}

To generate an ultraspherical window with $\mu$ and $N$ fixed and a prescribed null-to-null half width of $\omega_{n} \mathrm{rad} / \mathrm{s}$, one can select the parameter $x_{\mu}$ appropriately. This can be accomplished by calculating $x_{\mu}$ using the expression

$$
x_{\mu}=\frac{x_{N-1,1}^{(\mu)}}{\cos \left(\omega_{n} / 2\right)}
$$

where the zero $x_{N-1,1}^{(\mu)}$ can be found using Algorithm 1 with $l=1, \lambda=\mu, n=N-1$, and $\varepsilon=10^{-6}$.

\subsection{Main-lobe width}

To generate an ultraspherical window with $\mu$ and $N$ fixed and a prescribed main-lobe half width of $\omega_{r} \mathrm{rad} / \mathrm{s}$, one can select the parameter $x_{\mu}$ appropriately. This can be accomplished by calculating $x_{\mu}$ using the expression

$$
x_{\mu}=\frac{x_{a}}{\cos \left(\omega_{r} / 2\right)},
$$

where $x_{a}$ is defined by $C_{N-1}^{\mu}\left(x_{a}\right)=\operatorname{msgn}(\mu) \cdot \max (a, b)$ as identified in Figure 2. Parameter $x_{a}$ is found through a threestep process. First, the zero $x_{N-2,1}^{(\mu+1)}$ is found using Algorithm 1 with $l=1, \lambda=\mu+1, n=N-2$, and $\varepsilon=10^{-6}$, and then the parameter $a=\left|C_{N-1}^{\mu}\left(x_{N-2,1}^{(\mu+1)}\right)\right|$ is calculated. Second, the zero $x_{N-2, \text { rnd }[(N-2) / 2]}^{(1+1)}$ is found using Algorithm 1 with $l=\operatorname{rnd}[(N-2) / 2], \lambda=\mu+1, n=N-2$, and $\varepsilon=10^{-6}$, and then the parameter $b=\left|C_{N-1}^{\mu}\left(x_{N-2, \operatorname{rnd}[(N-2) / 2]}^{(\mu+1)}\right)\right|$ is calculated. Third, since $\operatorname{mggn}(\mu) \cdot \max (a, b)=C_{N-1}^{\mu}\left(x_{a}\right)$ as seen in Figure 2, parameter $x_{a}$ is found using a modified version of Algorithm 1 where (13) is replaced by

$$
y_{k+1}=y_{k}-\frac{C_{n}^{\lambda}\left(y_{k}\right)-\operatorname{msgn}(\mu) \cdot \max (a, b)}{2 \lambda C_{n-1}^{\lambda+1}\left(y_{k}\right)}
$$

and the starting point given in (12) is replaced by $y_{1}=1$. Instead of finding the largest zero of $f(x)=C_{n}^{\mu}(x)$, the modified algorithm finds the largest zero of $f(x)=C_{n}^{\mu}(x)-$ $\operatorname{msgn}(\mu) \cdot \max (a, b)$, which is parameter $x_{a}$. In the modified algorithm, $l=1, \lambda=\mu, n=N-1$, and $\varepsilon=10^{-6}$.

\subsection{Ripple ratio}

To generate an ultraspherical window with $\mu$ and $N$ fixed and a prescribed ripple ratio $r$, one can select the parameter $x_{\mu}$ appropriately. The parameter $x_{\mu}$ is found through a threestep process. First, the zero $x_{N-2,1}^{(\mu+1)}$ is found using Algorithm 1 with $l=1, \lambda=\mu+1, n=N-2$, and $\varepsilon=10^{-6}$ and then the parameter $a=\left|C_{N-1}^{\mu}\left(x_{N-2,1}^{(\mu+1)}\right)\right|$ is calculated. Second, the zero $x_{N-2, \text { rnd }[(N-2) / 2]}^{(\mu+1)}$ is found using Algorithm 1 with $l=\operatorname{rnd}[(N-2) / 2], \lambda=\mu+1, n=N-2$, and $\varepsilon=10^{-6}$, and then the parameter $b=\left|C_{N-1}^{\mu}\left(x_{N-2, \operatorname{rnd}[(N-2) / 2]}^{(\mu+1)}\right)\right|$ is calculated. Third, the parameter $x_{\mu}$ is found using a modified version of Algorithm 1 where (13) is replaced by

$$
y_{k+1}=y_{k}-\frac{C_{n}^{\lambda}\left(y_{k}\right)-\operatorname{msgn}(\mu) \cdot \max (a, b) / r}{2 \lambda C_{n-1}^{\lambda+1}\left(y_{k}\right)}
$$

and the starting point given in (12) is replaced by

$$
y_{1}=\cosh \left[\frac{1}{N-1} \cosh ^{-1}\left(\frac{1}{r}\right)\right] .
$$

Instead of finding the largest zero of $f(x)=C_{n}^{\mu}(x)$, the modified algorithm finds the largest zero of $f(x)=C_{n}^{\mu}(x)-$ $\operatorname{msgn}(\mu) \cdot \max (a, b) / r$ which is the parameter $x_{\mu}$. In the modified algorithm $l=1, \lambda=\mu, n=N-1$, and $\varepsilon=10^{-6}$.

\section{PREDICTION OF $N$}

In some applications designers may be able to choose the window length $N$. In such applications, the extra degree of freedom allows for more flexible window designs to be obtained. Specifically, solutions that are required to meet both a prescribed ripple ratio and width characteristic are possible. In this section, an empirical equation is proposed that predicts the ultraspherical window length $N$ required to achieve a prescribed side-lobe roll-off ratio, ripple ratio, and mainlobe width simultaneously. 


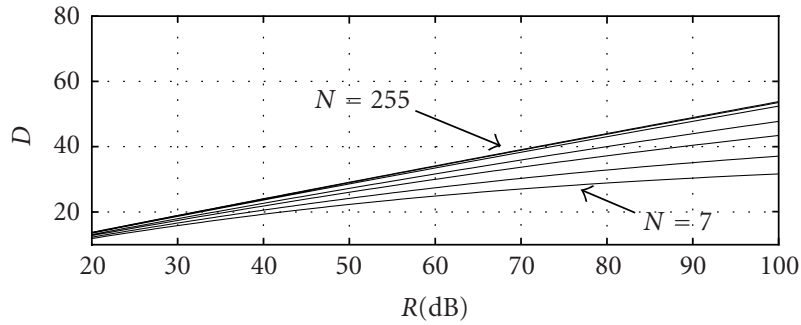

(a)

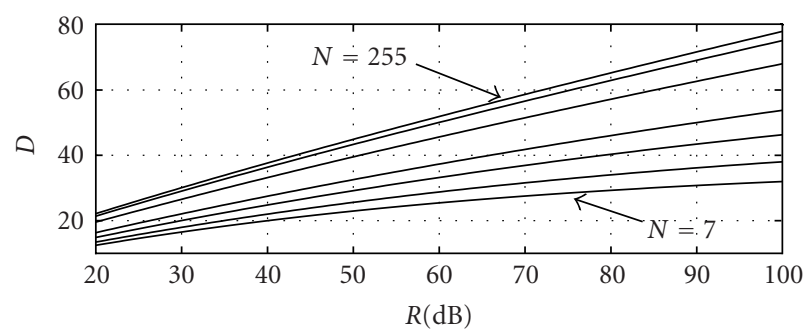

(b)

Figure 3: Performance factor $D$ versus $R$ in $\mathrm{dB}$ for windows of length $N=7,9,13,19,51,127$, and 255 for values of (a) $\mu=1$ and (b) $\mu=10$.

To obtain an equation for $N$, we employ the performance factor [18]

$$
D=2 \omega_{r}(N-1)
$$

which is used to give a normalized width that is approximately independent of $N$. Rearranging (34), an expression for $N$ is obtained as

$$
N \geq \frac{D}{2 \omega_{r}}+1
$$

where $N$ is rounded up to the nearest integer. From (35), it becomes clear that $N$ can be predicted by obtaining an accurate approximation of $D$.

\subsection{Measurements and tendencies of $D$}

To obtain realistic data for the approximation of $D$, windows of length $N=7,9,13,19,51,127$, and 255 were designed to cover the range $20 \leq R \leq 100$ in $\mathrm{dB}$ for the parameter range $-0.9999 \leq \mu \leq 10$. Figure 3 shows plots of $D$ versus $R$ in $\mathrm{dB}$ for the two cross-sections $\mu=1$ and 10. The plots tend to be quadratic and are representative for the range $-0.9999 \leq \mu \leq 10$ considered in this paper. Note the approximately linear behavior for $N=255$ indicating the independence of the performance factor $D$ with respect to $N$ for large $N$, which agrees with previous observations concerning the performance factor $D$ [18].

\subsection{Data-fitting procedure}

Before approximating $D$, the allowable error in the datafitting procedure must be determined. From (35), we note that for $N \gg 1$ a per-unit error in $D$ gives approximately the
TABLE 2: Model coefficients $a_{i j k}$ in (37) $(S>0)$.

\begin{tabular}{r|l|rrr}
\hline$i$ & $j$ & \multicolumn{1}{c}{$k=0$} & $k=1$ & \multicolumn{1}{c}{$k=2$} \\
\hline \multirow{4}{*}{0} & 0 & $2.699 \mathrm{E}+0$ & $1.824 \mathrm{E}-1$ & $-1.125 \mathrm{E}-1$ \\
& 1 & $4.650 \mathrm{E}-1$ & $-1.450 \mathrm{E}-2$ & $-1.607 \mathrm{E}-2$ \\
& 2 & $-6.273 \mathrm{E}-5$ & $2.681 \mathrm{E}-4$ & $-1.263 \mathrm{E}-4$ \\
\hline \multirow{4}{*}{1} & 0 & $2.657 \mathrm{E}-2$ & $8.293 \mathrm{E}-2$ & $-6.312 \mathrm{E}-2$ \\
& 1 & $1.719 \mathrm{E}-3$ & $1.846 \mathrm{E}-3$ & $7.488 \mathrm{E}-5$ \\
& 2 & $-4.610 \mathrm{E}-6$ & $-1.801 \mathrm{E}-5$ & $2.406 \mathrm{E}-6$ \\
\hline \multirow{3}{*}{2} & 0 & $-7.012 \mathrm{E}-5$ & $3.882 \mathrm{E}-4$ & $-1.703 \mathrm{E}-3$ \\
& 1 & $-5.568 \mathrm{E}-6$ & $7.549 \mathrm{E}-6$ & $1.153 \mathrm{E}-5$ \\
& 2 & $2.451 \mathrm{E}-8$ & $-6.588 \mathrm{E}-8$ & $1.139 \mathrm{E}-8$ \\
\hline
\end{tabular}

TABLE 3: Model coefficients $a_{i j k}$ in $(37)(S<0)$.

\begin{tabular}{r|r|rrr}
\hline$i$ & $j$ & \multicolumn{1}{c}{$k=0$} & $k=1$ & \multicolumn{1}{c}{$k=2$} \\
\hline \multirow{4}{*}{0} & 0 & $2.700 \mathrm{E}-0$ & $1.699 \mathrm{E}-1$ & $-1.126 \mathrm{E}-1$ \\
& 1 & $4.648 \mathrm{E}-1$ & $-1.321 \mathrm{E}-2$ & $-1.646 \mathrm{E}-2$ \\
& 2 & $-6.200 \mathrm{E}-5$ & $2.593 \mathrm{E}-4$ & $-1.230 \mathrm{E}-4$ \\
\hline \multirow{4}{*}{1} & 0 & $-2.214 \mathrm{E}-1$ & $1.095 \mathrm{E}-1$ & $-5.410 \mathrm{E}-2$ \\
& 1 & $-2.066 \mathrm{E}-3$ & $1.183 \mathrm{E}-3$ & $5.045 \mathrm{E}-4$ \\
& 2 & $1.723 \mathrm{E}-5$ & $-1.617 \mathrm{E}-5$ & $1.242 \mathrm{E}-6$ \\
\hline \multirow{3}{*}{2} & 0 & $-2.016 \mathrm{E}-3$ & $-6.856 \mathrm{E}-3$ & $5.755 \mathrm{E}-3$ \\
& 1 & $-1.646 \mathrm{E}-5$ & $1.248 \mathrm{E}-4$ & $-9.390 \mathrm{E}-5$ \\
& 2 & $3.492 \mathrm{E}-7$ & $-1.409 \mathrm{E}-6$ & $8.638 \mathrm{E}-7$ \\
\hline
\end{tabular}

same per-unit error in $N$, that is,

$$
\frac{\Delta D}{D}=\frac{\Delta(N-1)}{N-1}=\frac{\Delta N}{N-1} \approx \frac{\Delta N}{N} .
$$

For example, if $N=127$ and a relative error in $D$ of $1.00 \%$ is assumed, that is, $\Delta D / D=0.01$, then an equivalent error of 1.26 samples in $N$ occurs. Errors of this magnitude have been considered acceptable in the past [18] as $N$ may be in error by at most 1 or 2 and only for high window lengths. Thus, the relative error $\Delta D / D \leq 0.01$ is sought throughout the approximation procedure.

A general quadratic model was used for the approximation of $D$ as a function of $S$ in $\mathrm{dB}, R$ in $\mathrm{dB}$, and the main-lobe half width $\omega_{r}$. Such a model takes the form

$$
D_{\text {aprx }}\left(S, R, \omega_{r}\right)=\sum_{i=0}^{2} \sum_{j=0}^{2} \sum_{k=0}^{2} a_{i j k} \phi(i, j, k),
$$

where $\phi(i, j, k)=(S / 20)^{i} R^{j} \omega_{r}^{k}$. The coefficients $a_{i j k}$ were found through a linear least-squares solution of the overdetermined system of sampled data points $\left\{S, R, \omega_{r}, D\right\}$ where $D$ is the dependent variable.

Two separate sets of 27 coefficients were found for the ranges $0 \leq S \leq 60$ and $-20 \leq S \leq 0$ given in $\mathrm{dB}$ and are provided in Tables 2 and 3, respectively. Two sets were required to produce accurate solutions due the nature of $D$ and its relation to positive and negative $S$ values. Figure 4 shows plots of the relative error of the predicted $D$ versus $R$ for various 


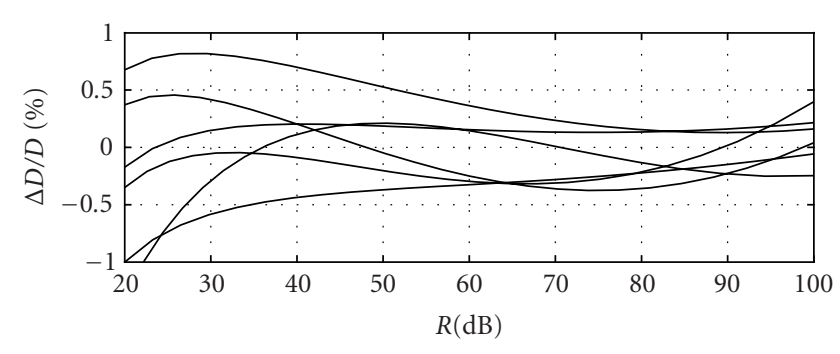

(a)

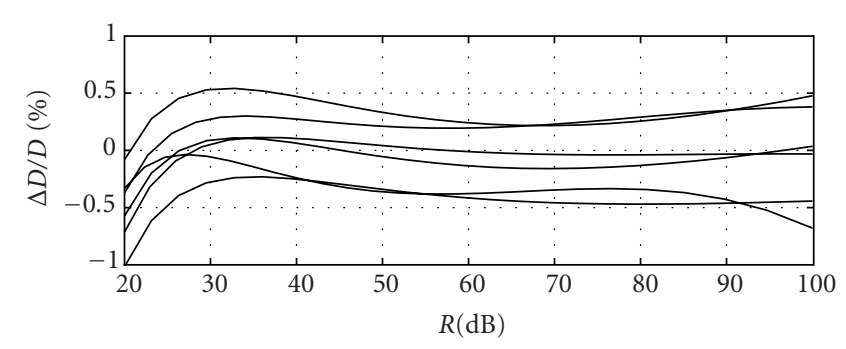

(b)

FIgURE 4: Relative error of predicted $D, \Delta D / D$, in percent versus $R$ in $\mathrm{dB}$ for window lengths $N=7,9,13,19,51,127$, and 255 over the cross sections (a) $\mu=1$ and (b) $\mu=-0.6$.

window lengths over the cross sections $\mu=1$ and -0.6 . The mean of the absolute relative error for the approximations given by Tables 2 and 3 is 0.2874 and $0.2266 \%$, respectively. Less error occurs for the coefficients in Table 3 because the approximation was performed over a smaller range of $S$ than that used for Table 2. The absolute relative error exceeds $1.0 \%$ only for small values of $R$ less than 20 and large values of $R$ greater than 100 .

In an attempt to reduce the number of approximation model coefficients, the quadratic model

$$
D_{\text {aprx }}\left(S, R, \omega_{r}\right)=\sum_{i=0} \sum_{j=0}^{l} \sum_{k=0} a_{i j k} \phi(i, j, k),
$$

where

$$
l=i+j+k \leq 2
$$

was investigated which yields 10 coefficients as opposed to 27. Using the same data fitting technique as before, the mean of the absolute relative error for the entire approximation was found to be $1.0911 \%$. In $70 \%$ of the predictions, the absolute error was less than $1.0 \%$.

On the basis of the above experiments, $N$ can be accurately predicted using the formula

$$
N=\operatorname{int}\left[\frac{D_{\text {aprx }}\left(S, R, \omega_{r}\right)}{2 \omega_{r}}+1.5\right],
$$

where $D_{\text {aprx }}$ is given by the 27 -term approximation model described in (37) using the coefficients provided in Tables 2 and 3.

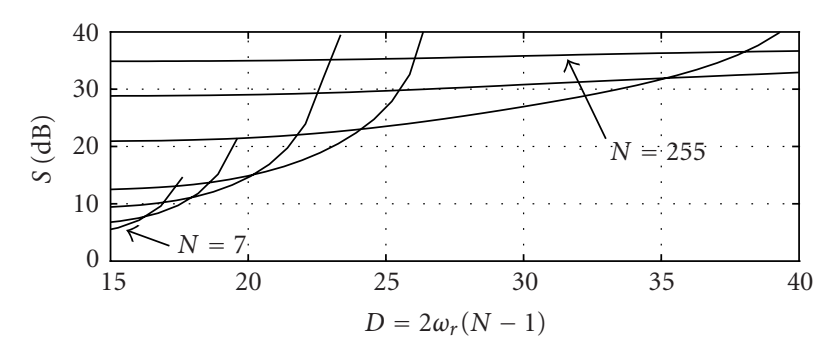

(a)

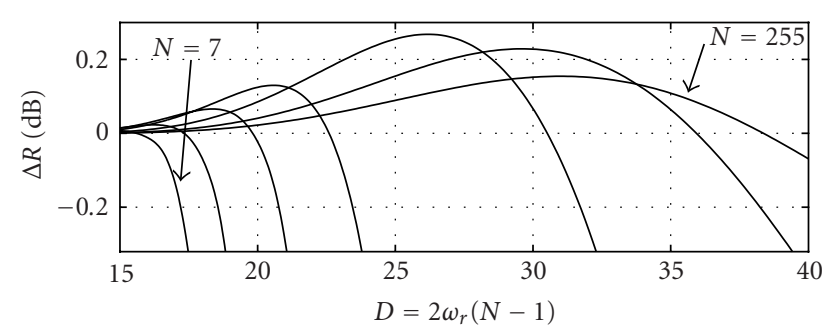

(b)

FIGURE 5: (a) Side-lobe roll-off ratio in $\mathrm{dB}$ for Kaiser windows of length $N=7,9,13,19,51,127$, and 255. (b) Change in $R$ in $\mathrm{dB}$ provided by ultraspherical windows of the same length that were designed to match the Kaiser windows' side-lobe roll-off ratio and main-lobe width.

The same process can be used to predict $N$ for other width characteristics such as the null-to-null or $3 \mathrm{~dB}$ widths.

\section{COMPARISON WITH OTHER WINDOWS}

For a fixed window length, two-parameter windows such as the Kaiser, Saramäki, and Dolph-Chebyshev windows can control the ripple ratio. The three-parameter ultraspherical window can control the ripple ratio as well as the side-lobe roll-off ratio. For comparison's sake, ultraspherical windows of the same length were designed to achieve the side-lobe roll-off ratio and main-lobe width produced by the Kaiser window, for values of the Kaiser-window parameter $\alpha$ in the range $[1,10]$, and the resulting ripple ratios for the two window families were measured and compared. The DolphChebyshev and Saramäki windows were excluded from the comparison because these windows are special cases of the ultraspherical window that can be readily obtained by fixing parameter $\mu$ to 0 and 1, respectively. Figure 5a shows plots of the side-lobe roll-off ratio in $\mathrm{dB}$ obtained for Kaiser windows of varying length versus $D=2 \omega_{r}(N-1)$ and Figure $5 \mathrm{~b}$ shows a plot of $\Delta R$ which is defined as

$$
\Delta R=R_{U}-R_{K},
$$

where $R_{U}$ and $R_{K}$ are the values of $R$ for ultraspherical and Kaiser windows, respectively, in $\mathrm{dB}$ for the same length, side roll-off ratio, and main-lobe width. As can be seen, the ultraspherical window offers a reduced ripple ratio for low values of $D$ whereas the Kaiser window gives better results for large values of $D$. Thus, for a given value of $N$, there is a 


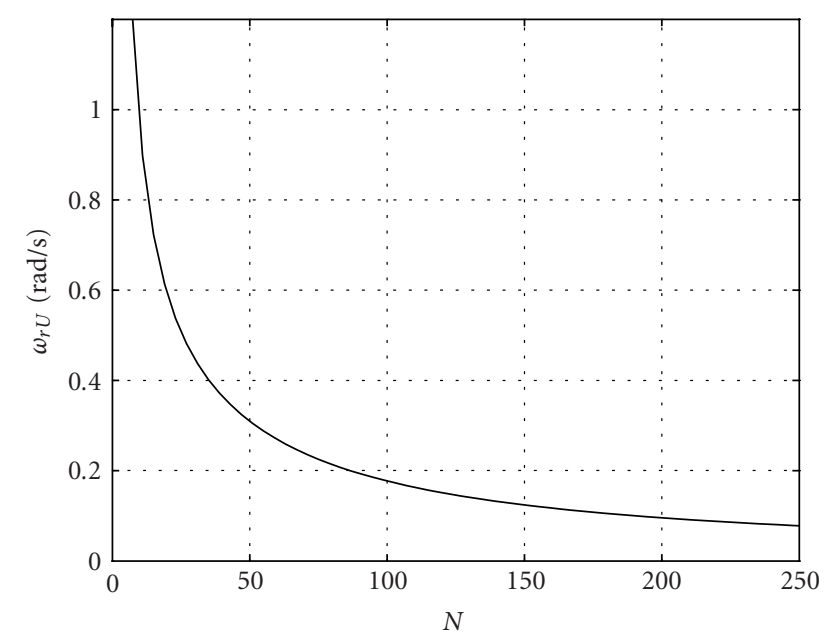

FIgURE 6: Values of the main-lobe half width that achieve the same ripple ratio for both the Kaiser and ultraspherical windows.

TABLe 4: Model coefficients for $\omega_{r U}$ in (42).

\begin{tabular}{l|cccc}
\hline$N_{L} N_{H}$ & $a$ & $b$ & $c$ & $d$ \\
\hline 1025 & $-1.149 \mathrm{E}-4$ & $7.855 \mathrm{E}-3$ & $-1.935 \mathrm{E}-1$ & $2.238 \mathrm{E}+0$ \\
2580 & $-1.495 \mathrm{E}-6$ & $3.208 \mathrm{E}-4$ & $-2.554 \mathrm{E}-2$ & $9.692 \mathrm{E}-1$ \\
80250 & $-2.520 \mathrm{E}-8$ & $1.679 \mathrm{E}-5$ & $-4.096 \mathrm{E}-3$ & $4.451 \mathrm{E}-1$ \\
\hline
\end{tabular}

corresponding main-lobe half width, say $\omega_{r U}$, for which the ultraspherical window gives a better ripple ratio than the Kaiser window. For main-lobe half widths that are larger than $\omega_{r U}$, the Kaiser window gives a smaller ripple ratio. A plot of $\omega_{r U}$ versus $N$ is shown in Figure 6. From this plot, a formula can be obtained for $\omega_{r U}$ as

$$
\omega_{r U}=a N^{3}+b N^{2}+c N+d \quad \text { for } N_{L} \leq N \leq N_{H},
$$

where the coefficients are presented in Table 4 . In effect, if the point $\left[N, \omega_{r}\right]$ is located below the curve in Figure 6 , the ultraspherical window is preferred, and if it is located above the curve, the Kaiser window is preferred.

\section{EXAMPLES}

Example 1. For $N=51$, generate the ultraspherical windows that will yield $S=20 \mathrm{~dB}$ for (a) $\omega_{r}=0.25 \mathrm{rad} / \mathrm{s}$ and (b) $\omega_{n}=$ $0.25 \mathrm{rad} / \mathrm{s}$.

Figure 7 shows the amplitude spectrums of the windows obtained. Both designs meet the prescribed specifications and produced (a) $R=42.97 \mathrm{~dB}$ and (b) $R=40.85 \mathrm{~dB}$. For both designs, the minimization of (27) resulted in $\mu=$ 0.9517 and (30) and (29) gave (a) $x_{\mu}=1.0067$ and (b) $x_{\mu}=1.0060$, respectively.

Example 2. For $N=51$, generate the ultraspherical windows that will yield $R=50 \mathrm{~dB}$ for (a) $S=-10 \mathrm{~dB}$ and (b) $S=$ $30 \mathrm{~dB}$.

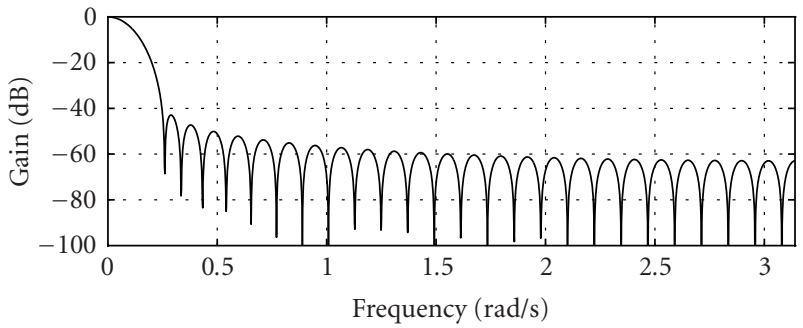

(a)

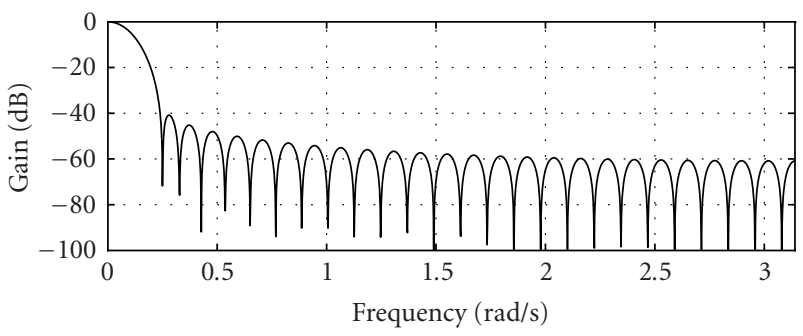

(b)

Figure 7: Ultraspherical window amplitude spectrums for $N=51$ yielding $S=20 \mathrm{~dB}$ for (a) $\omega_{r}=0.25 \mathrm{rad} / \mathrm{s}$ and (b) $\omega_{n}=0.25 \mathrm{rad} / \mathrm{s}$ (Example 1).

Figure 8 shows the amplitude spectrums of the windows obtained. Both designs met the prescribed specifications and produced main-lobe widths of (a) $\omega_{r}=0.2783 \mathrm{rad} / \mathrm{s}$ and (b) $\omega_{r}=0.2975 \mathrm{rad} / \mathrm{s}$. Minimizing (27) resulted in (a) $\mu=$ -0.3914 and (b) $\mu=1.5151$ and the procedure described in Section 4.4 gave (a) $x_{\mu}=1.0107$ and (b) $x_{\mu}=1.0091$.

Example 3. Predict the required window length $N$ and generate the ultraspherical windows that will yield $\omega_{r}=0.2 \mathrm{rad} / \mathrm{s}$ and $R \geq 60 \mathrm{~dB}$ for (a) $S=10 \mathrm{~dB}$ and (b) $S=-10 \mathrm{~dB}$.

A consequence of rounding $N$ up to the nearest integer is that one prescribed spectral characteristic is oversatisfied. For the method presented in this paper, one will always achieve $S$ and either $\omega_{r}$ or $R$ to a high degree of precision by using either (30) or the procedure described in Section 4.4 as appropriate to calculate parameter $x_{\mu}$. In this example, we oversatisfy $R$ by using (30). Figure 9 shows the amplitude spectrums of the windows obtained. Both designs meet the prescribed characteristics and oversatisfied $R$ by (a) $0.47 \mathrm{~dB}$ and (b) $0.41 \mathrm{~dB}$. Using the prediction formula given in (40), the window lengths required to achieve the prescribed characteristics were (a) $N=81$ and (b) $N=83$. Minimizing (27) resulted in (a) $\mu=0.3756$ and (b) $\mu=-0.3378$ and (30) gave (a) $x_{\mu}=1.0049$ and (b) $x_{\mu}=1.0053$.

To examine the accuracy of the window length prediction formula, windows were designed to achieve the same prescribed characteristics with window lengths taken to be one less than predicted by (40), that is, for (a) $N-1=$ 80 and (b) $N-1=82$. Figure 10 shows the amplitude spectrums obtained for $N$ and $N-1$ in the critical area near the main-lobe edge. All windows were found to satisfy the $S$ and $\omega_{r}$ specifications; however, both windows 


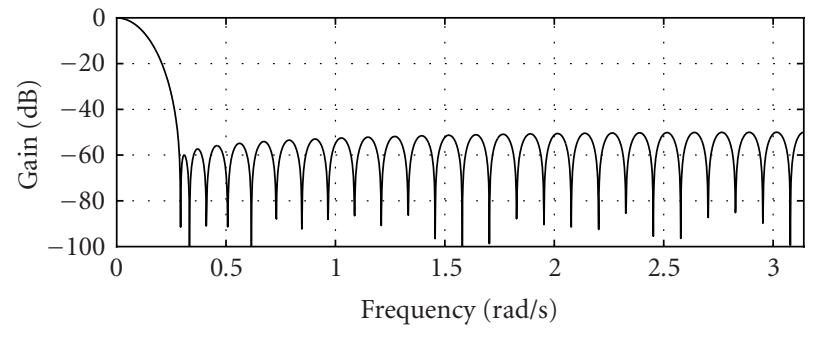

(a)

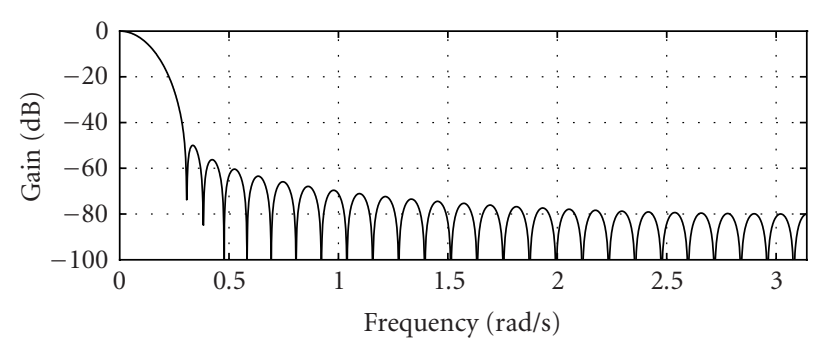

(b)

Figure 8: Ultraspherical window amplitude spectrums for $N=51$ yielding $R=50 \mathrm{~dB}$ for (a) $S=-10 \mathrm{~dB}$ and (b) $S=30 \mathrm{~dB}$ (Example 2).

of the reduced length fell short of $R \geq 60 \mathrm{~dB}$ by (a) $0.35 \mathrm{~dB}$ and (b) $0.51 \mathrm{~dB}$. The results demonstrate the accuracy of (40) in predicting the lowest value of $N$ needed to achieve the set of prescribed spectral characteristics simultaneously.

Example 4. For $N=101$, generate Kaiser and ultraspherical windows that will yield (a) $R=50 \mathrm{~dB}$ and (b) $R=70 \mathrm{~dB}$ and compare the results obtained.

The required Kaiser-window parameter $\alpha$ for (a) and (b) can be predicted using the formula [19]

$$
\alpha= \begin{cases}0, & R \leq 13.26, \\ 0.76609(R-13.26)^{0.4} & 13.26<R \leq 60 \\ +0.09834(R-13.26), & \\ 0.12438(R+6.3), & 60<R \leq 120,\end{cases}
$$

as $\alpha=6.8514$ and 9.4902 producing main-lobe half widths of $\omega_{r}=0.1462$ and $0.1964 \mathrm{rad} / \mathrm{s}$, respectively. Ultraspherical windows were designed to achieve the same side-lobe roll-off ratio and main-lobe widths as the Kaiser windows measured as (a) $S=29.19 \mathrm{~dB}$ and (b) $S=32.02 \mathrm{~dB}$. Minimizing (27) resulted in (a) $\mu=1.0976$ and (b) $\mu=1.2165$, and the procedure described in Section 4.4 gave (a) $x_{\mu}=1.0023$ and (b) $x_{\mu}=1.0044$. The difference in $R$ was (a) $\Delta R=0.2236$ and (b) $\Delta R=-0.4496 \mathrm{~dB}$. Thus, the ultraspherical window gives a better ripple ratio in (a) and the Kaiser window gives a better ripple ratio in (b) in agreement with (42).

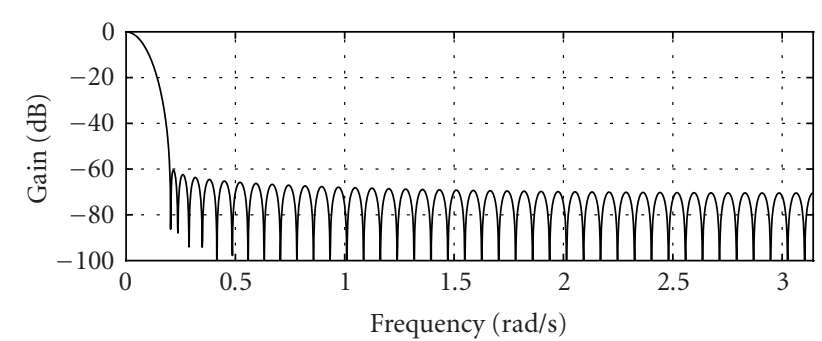

(a)

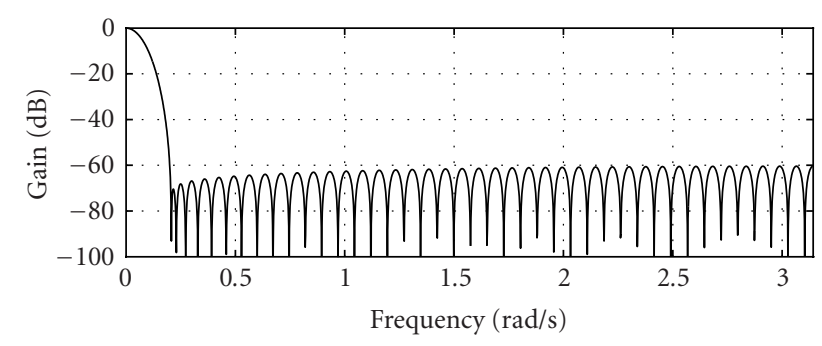

(b)

FIGURE 9: Ultraspherical window amplitude spectrums yielding $\omega_{R}=0.2 \mathrm{rad} / \mathrm{s}$ and $R \geq 60 \mathrm{~dB}$ for (a) $S=10 \mathrm{~dB}$ and (b) $S=-10 \mathrm{~dB}$ (Example 3(a)).

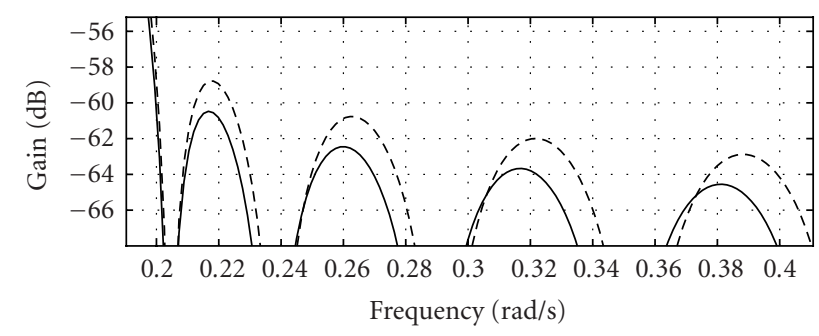

(a)

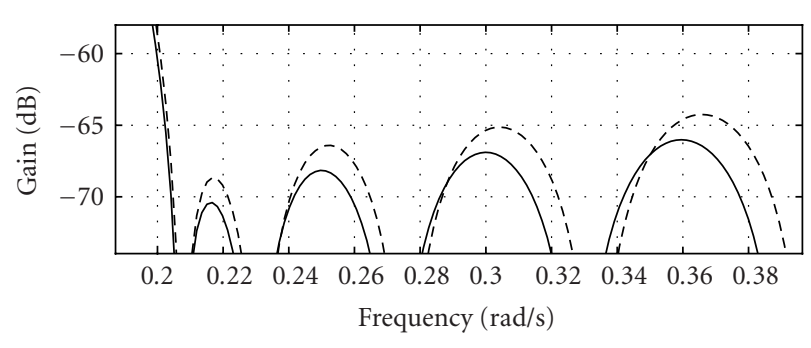

(b)

FIGURE 10: Ultraspherical window amplitude spectrums for predicted $N$ (solid line) and predicted $N-1$ (dashed line) yielding $\omega_{R}=0.2 \mathrm{rad} / \mathrm{s}$ and $R \geq 60 \mathrm{~dB}$ for (a) $S=10 \mathrm{~dB}$ and (b) $S=-10 \mathrm{~dB}$ (Example 3(b)).

\section{APPLICATIONS}

The ultraspherical window function has been presented in terms of its spectral characteristics to facilitate its use for a diverse range of applications. The flexibility provided by our ability to control the side-lobe roll-off ratio has enabled us 
to develop a method for the design of FIR filters that satisfy prescribed specifications, which leads to improved filter specifications relative to the Kaiser window method [20, 21]. In this section, two other window applications, beamforming and image processing, are presented to illustrate the benefits obtained by exercising the proposed methods flexibility.

\subsection{Beamforming}

In radar, ocean acoustics, and ultrasonics it is necessary to design antenna or transducer systems with specific directivity properties, that is, for point-to-point communication systems, a high gain in one direction with low gain in all other directions is considered desirable. Known as beamforming, this activity shapes the radiation pattern (or beam) of a transmitted signal so that most of its energy propagates towards the intended receiver or target. Similarly, when receiving signals, the receiver sensitivity (or beam) can be directed towards the transmitter or source to receive the maximum signal strength possible. Directing and focusing signal energy in this fashion leads to the rejection of interference from other sources and to reduced power requirements for transmitter and receiver power, which in turn provides cost savings.

One practical and common antenna/transducer configuration is the linear array, which is characterized by having all its radiating elements positioned in a straight line. Linear arrays can consist of one continuous radiating element or a number of individual discrete elements. Generally, discrete elements are favored because of their capability to dynamically change the directivity properties of the array. The array factor (AF) is used to describe an array's directivity properties. For a broadside array of length $N$ with amplitude excitations for each isotropic element being symmetrical about the center of the array, the AF is given by [22]

$$
\operatorname{AF}(\theta)= \begin{cases}\sum_{n=1}^{r} a_{n}^{\prime} \cos [(2 n-1) u] & \text { for odd } N \\ \sum_{n=1}^{r} a_{n} \cos [2(n-1) u] & \text { for even } N\end{cases}
$$

where

$$
\begin{aligned}
& u=\text { the spatial frequency (degrees } / \mathrm{m} \text { ) } \\
& =\frac{\pi d}{\lambda} \cos \theta, \\
& \theta=\text { the bearing angle (degrees), } \\
& d=\text { the spacing between elements (m), } \\
& \lambda=\text { the wavelength of the signal (m), } \\
& a_{n}=\text { the excitation coefficients or currents (A), } \\
& a_{n}^{\prime}= \begin{cases}a_{n}, & n \neq 1, \\
\frac{1}{2} a_{n}, & n=1,\end{cases} \\
& r= \begin{cases}\frac{N+1}{2} & \text { for odd } N \\
\frac{N}{2} & \text { for even } N\end{cases}
\end{aligned}
$$

The relationship between $\operatorname{AF}(\theta)$ and $a_{n}$ is analogous to the relationship between $W\left(e^{j \omega T}\right)$ and $w(n T)$. This similarity allows window design techniques to be applied directly to the design of antenna arrays. As in window designs, the trade-off between the main-lobe width and the side-lobe level of the $\mathrm{AF}$ is of primary importance. In the uniform array the excitation coefficients are all equal, as in the rectangular window, and hence the main-lobe width of the AF is narrow and sidelobe levels are large. At the other extreme, the binomial array's AF has no side lobes but has of a large main-lobe width. Practical difficulties also arise with the implementation of the binomial array because the difference between excitation coefficients can be considerable leading to disparate current requirements. The Dolph-Chebyshev array, which offers an adjustable trade-off between the main-lobe width and side-lobe level, overcomes the implementation difficulties associated with the binomial array and is generally accepted as being a practical compromise between the uniform and binomial arrays. The Dolph-Chebyshev array's AF suggests it is best used when no prior knowledge of the interference sources is available, that is, the likelihood of interference is equal at all locations. However, if the general location of interference sources can be identified, not much can be done to compensate with the Dolph-Chebyshev array.

One solution could be to use the more flexible three-parameter ultraspherical weights instead of the twoparameter Dolph-Chebyshev weights, in which case the excitation coefficients are given by

$$
a_{n}=w[(r+n-1) T] \text { for } n=1,2, \ldots, r,
$$

where $w(n T)$ are the coefficients provided by (6) resulting in

$$
\operatorname{AF}(\theta)=C_{N-1}^{\mu}\left(x_{\mu} \cos u\right)
$$

This is equivalent to the amplitude function of the ultraspherical window given in (16) with the substitution $u=$ $\omega T / 2$. Similarly, all the techniques developed in this paper are easily transferable to customizing the directivity properties of linear arrays. Fair comparisons between the two AFs can be made by designing ultraspherical and Dolph-Chebyshev arrays of the same length and the same null-to-null width, and then measuring the ripple ratios. To accomplish this, we make $\cos \left(\omega_{n} / 2\right)$ in (29) equal for both the Dolph-Chebyshev and ultraspherical arrays, which yields the relation

$$
\frac{x_{N-1,1}^{(\mu)}}{x_{\mu}}=\frac{x_{N-1,1}^{(0)}}{x_{0}}=\frac{\cos [\pi / 2(N-1)]}{x_{0}},
$$

where $x_{0}$ is given by (19). Substituting and rearranging yields the closed-form expression for the ripple ratio

$$
r=\frac{1}{\cosh \left\{(N-1) \cosh ^{-1}\left[\left(x_{\mu} / x_{N-1,1}^{(\mu)}\right) \cos (\pi / 2(N-1))\right]\right\}}
$$




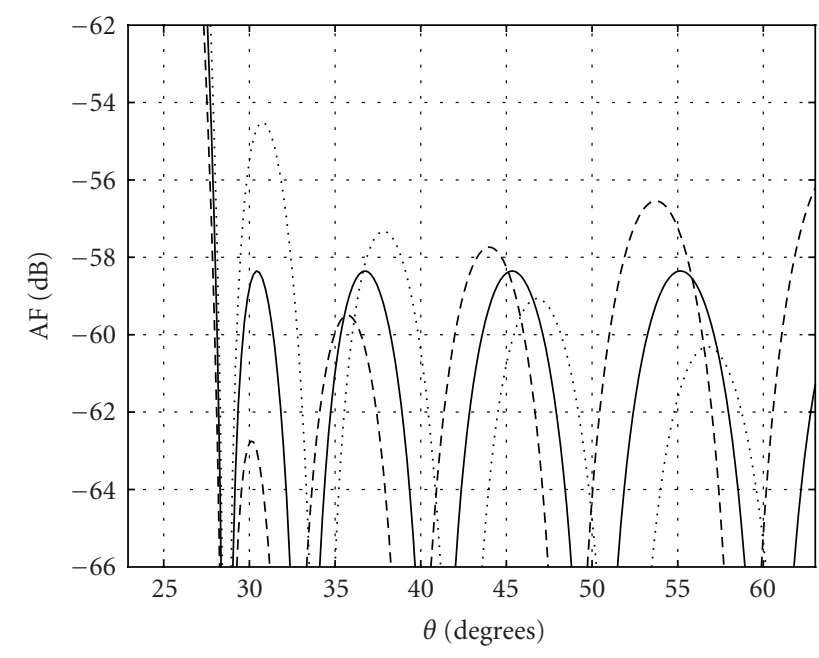

FIgURE 11: AF for the ultraspherical array of length $N=31$ and $\theta_{n}=28.6479$ degrees for the cases where $S=0 \mathrm{~dB}$ (solid line), $S=$ $-10 \mathrm{~dB}$ (dashed line), and $S=10 \mathrm{~dB}$ (dotted line).

that the Dolph-Chebyshev array of the same length and null-to-null width would produce compared to an ultraspherical array. This expression can be used to judge how much ripple ratio is sacrificed to attain a given side-lobe pattern.

Figure 11 shows enlarged plots around the first null of three ultraspherical arrays designed with $N=31, \omega_{n}=$ $0.5 \mathrm{rad} / \mathrm{s}\left(\theta_{n}=28.6479\right.$ degrees $)$, and $S=-10,0$, and $10 \mathrm{~dB}$. The first side-lobe peak is $4.38 \mathrm{~dB}$ less for the case $S=-10 \mathrm{~dB}$ and $3.84 \mathrm{~dB}$ more for the case $S=10$ relative to the peak for the case $S=0$ (i.e., the Dolph-Chebyshev array). On the other hand, the furthest side-lobe peak (not shown) is $5.62 \mathrm{~dB}$ more for $S=-10$ and $6.16 \mathrm{~dB}$ less for $S=10 \mathrm{~dB}$ relative to the peak for $S=0$. The ripple ratio for the Dolph-Chebyshev array is given by (49) as $-58.35 \mathrm{~dB}$. An important observation is that the positioning of the second null weighs heavily on the amplitude of the first side lobe, which, in turn, is very important in determining the amplitude of the remaining side lobes. To this extent, an alteration in the amplitude of the first side lobe greatly influences the amplitude of the remaining side lobes in an inverse fashion, that is, increasing the first side-lobe amplitude decreases most of the remaining side-lobe amplitudes. Experimental results indicate that the side lobe envelope of the ultraspherical array tends to cross that of the Dolph-Chebyshev array within the first three side lobes adjacent to the main lobe. In this respect, negative $S$ values are preferred to the DolphChebyshev array for narrowband interference sources that are confined to this region. Alternatively, positive $S$ values are preferred for interference sources that fall past this region. Using the methods proposed in this paper, antenna array designers are provided with an easy-to-use visual design approach for deciding what amount of trade-off between sidelobe pattern and ripple ratio is best for their particular situation.

\subsection{Image processing}

With the ever-expanding gamut of computer monitors, hand-held devices such as digital cameras and video recorders, and high-end medical imaging systems, consumers can often base purchasing decisions on a few key image quality measures. On such measure is an image's contrast ratio (CR) which, simply put, defines the difference in light intensity between the darkest black and brightest white shades within an image. A high CR allows one to discern detailed differences between colors producing a crisp and sharp image. On the other hand, a low CR results in a blurring or smearing effect producing an image with little clarity. A direct consequence of the CR measure is its effect on an imaging system's capability to detect low-contrast objects residing near high-contrast objects, which can be of the utmost importance in some medical imaging applications, for example, detecting cancerous tumors. Also, interpretation of an image's quality has been shown, through human trials, to be directly related to the CR measure [23].

A number of imaging systems such as synthetic aperture radar (SAR) [24], computerized tomography (CAT scans) [24], and charge-coupled device (CCD)-based X-rays [25] construct images by using two-dimensional windowed inverse DFTs on spatial frequency-domain data. For these systems CR tolerance is usually specified in terms of the worstcase spectral leakage of the window function used, which is directly related to the window's main-lobe to side-lobe energy ratio (MSR). Strictly speaking, the CR is defined as [26]

$$
\mathrm{CR}=\frac{E_{s}+E_{m}}{E_{s}}=1+\mathrm{MSR},
$$

where the side-lobe and main-lobe energies are given by

$$
\begin{gathered}
E_{s}=\int_{\omega_{r}}^{\pi}\left|W\left(e^{j \omega T}\right)\right|^{2} d \omega, \\
E_{m}=\int_{0}^{\omega_{r}}\left|W\left(e^{j \omega T}\right)\right|^{2} d \omega,
\end{gathered}
$$

respectively, and MSR $=E_{m} / E_{s}$. By referring to the window's spectral representation as the inner product of the Fourier kernel

$$
\mathbf{v}=\left[\begin{array}{lllll}
1 & e^{-j \omega T} & e^{-j 2 \omega T} & \cdots & e^{-j(N-1) \omega T}
\end{array}\right]
$$

with the window coefficient vector $\mathbf{w}$, that is, $W\left(e^{j \omega T}\right)=$ $\mathbf{w}^{T} \mathbf{v}$, the side-lobe energy $E_{s}$ can be expressed in the form

$$
E_{s}=\mathbf{w}^{T} \mathbf{Q} \mathbf{w},
$$

where

$$
\mathbf{Q}=\mathbf{Q}\left(\omega_{r}\right)=2 \int_{\omega_{r}}^{\pi} \mathbf{V} d \omega
$$




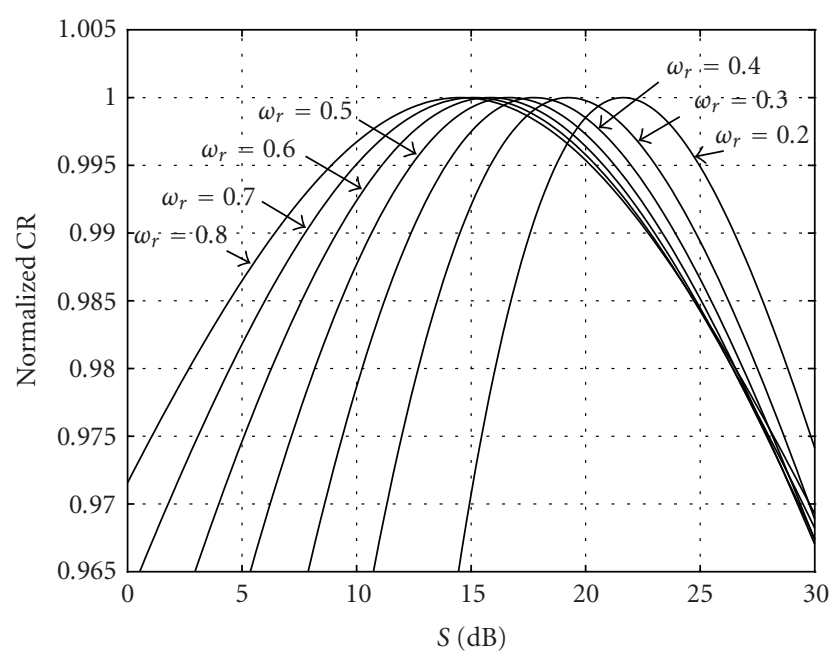

FIGURE 12: The normalized CR versus side-lobe roll-off ratio $S$ with various main-lobe half-width quantities for the ultraspherical window of length $N=31$.

and $\mathbf{V}=\mathbf{v v}^{*}$. The elements of $\mathbf{Q}$ are given by

$$
q(n, m)= \begin{cases}-\frac{\omega_{r}}{\pi} \operatorname{sinc}\left[\omega_{r}(m-n)\right] & \text { for } m \neq n, \\ 1-\frac{\omega_{r}}{\pi} & \text { for } m=n,\end{cases}
$$

where $\mathbf{Q}$ is a real, symmetric, positive-definite Toeplitz matrix. Using Parseval's theorem, the total energy is found as

$$
E_{t}=E_{m}+E_{s}=\mathbf{w}^{T} \mathbf{w}
$$

where a simple rearrangement yields the main-lobe energy $E_{m}$. Thus a window's CR can be calculated as

$$
\mathrm{CR}=\frac{\mathbf{w}^{T} \mathbf{w}}{\mathbf{w}^{T} \mathbf{Q} \mathbf{w}} .
$$

Using the flexible three-parameter ultraspherical window for the windowing operation, the side-lobe patterns can be easily adjusted to alter the energy contained in the side lobes and, consequently, the value of the CR measure. Figure 12 shows plots of the normalized CR versus the side-lobe rolloff ratio $S$ in $\mathrm{dB}$ for various main-lobe half-width quantities. The curves are convex with easily discernible global maximum values. As such, the ultraspherical window that possesses the maximum CR for a given window length $N$ and main-lobe width $\omega_{r}$ can be found through the appropriate selection of $S$. This can be accomplished by solving the onedimensional optimization problem

$$
\operatorname{minimize}_{S_{L} \leq S \leq S_{H}} F=-\mathrm{CR}=-\frac{\mathbf{w}^{T} \mathbf{w}}{\mathbf{w}^{T} \mathbf{Q} \mathbf{w}},
$$

where vector $\mathbf{w}$ is calculated using (6) and the techniques described in Sections 4.1 and 4.3, the $\mathbf{Q}$ matrix is calculated using (55), $S_{L}=0 \mathrm{~dB}$, and $S_{H}=30 \mathrm{~dB}$. For the example with $N=31$ and $\omega_{r}=0.4 \mathrm{rad} / \mathrm{s}$, the solution of (58) yields a maximum CR value of $41.01 \mathrm{~dB}$ occurring at $S=17.75 \mathrm{~dB}$. The corresponding parameters for the ultraspherical window are $\mu=1.0810$ and $x_{\mu}=1.0166$.

\section{CONCLUSIONS}

A method for the design of ultraspherical windows that achieves prescribed spectral characteristics has been proposed. The method comprises a collection of techniques that can be used to determine the independent parameters of the ultraspherical window such that a specified ripple ratio, main-lobe width, or null-to-null width along with a specified side-lobe roll-off ratio can be achieved. The Kaiser, Saramäki, and Dolph-Chebyshev two-parameter windows can achieve a specified ripple ratio and main-lobe width; however their side-lobe patterns cannot be controlled as in the proposed method. Experimental results have shown that the desired characteristics can be achieved with a high degree of precision. The ultraspherical window includes both the DolphChebyshev and Saramäki windows as particular cases and a difference in the performance of the ultraspherical and Kaiser windows has been identified, which depends critically on the required specifications. The paper has also shown that the proposed design method can be used to achieve improved performance in beamforming and image processing systems.

\section{REFERENCES}

[1] S. R. Seydnejad and R. I. Kitney, "Real-time heart rate variability extraction using the Kaiser window," IEEE Trans. on Biomedical Engineering, vol. 44, no. 10, pp. 990-1005, 1997.

[2] R. M. Rangayyan, Biomedical Signal Analysis: A Case-Study Approach, Wiley-IEEE Press, New York, NY, USA, 2002.

[3] S. He and J.-Y. Lu, "Sidelobe reduction of limited diffraction beams with Chebyshev aperture apodization," Journal of the Acoustical Society of America, vol. 107, no. 6, pp. 3556-3559, 2000.

[4] E. Torbet, M. J. Devlin, W. B. Dorwart, et al., "A measurement of the angular power spectrum of the microwave background made from the high Chilean Andes," The Astrophysical Journal, vol. 521, pp. L79-L82, 1999.

[5] B. Picard, E. Anterrieu, G. Caudal, and P. Waldteufel, "Improved windowing functions for Y-shaped synthetic aperture imaging radiometers," in Proc. IEEE International Geoscience and Remote Sensing Symposium (IGARSS '02), vol. 5, pp. 2756-2758, Toronto, Ont, Canada, June 2002.

[6] P. Lynch, "The Dolph-Chebyshev window: a simple optimal filter," Monthly Weather Review, vol. 125, pp. 655-660, 1997.

[7] T. Saramäki, "Finite impulse response filter design," in Handbook for Digital Signal Processing, S. K. Mitra and J. F. Kaiser, Eds., Wiley, New York, NY, USA, 1993.

[8] J. F. Kaiser, "Nonrecursive digital filter design using $I_{0}$-sinh window function.," in Proc. IEEE Int. Symp. Circuits and Systems (ISCAS '74), pp. 20-23, San Francisco, Calif, USA, April 1974.

[9] T. Saramäki, "A class of window functions with nearly minimum sidelobe energy for designing FIR filters," in Proc. IEEE Int. Symp. Circuits and Systems (ISCAS '89), vol. 1, pp. 359362, Portland, Ore, USA, May 1989.

[10] C. L. Dolph, "A current distribution for broadside arrays which optimizes the relationship between beamwidth and side-lobe level," Proc. IRE, vol. 34, pp. 335-348, June 1946.

[11] F. J. Harris, "On the use of windows for harmonic analysis with the discrete Fourier transform," Proceedings of the IEEE, vol. 66, no. 1, pp. 51-83, 1978.

[12] R. L. Streit, "A two-parameter family of weights for nonrecursive digital filters and antennas," IEEE Trans. Acoustics, Speech, and Signal Processing, vol. 32, no. 1, pp. 108-118, 1984. 
[13] A. G. Deczky, "Unispherical windows," in Proc. IEEE Int. Symp. Circuits and Systems (ISCAS '01), vol. 2, pp. 85-88, Sydney, NSW, Australia, May 2001.

[14] S. W. A. Bergen and A. Antoniou, "Generation of ultraspherical window functions," in XI European Signal Processing Conference, vol. 2, pp. 607-610, Toulouse, France, September 2002.

[15] M. Abramowitz and I. A. Stegun, Eds., Handbook of Mathematical Functions with Formulas, Graphs and Mathematical Tables, vol. 55 of National Bureau of Standards Applied Mathematics Series, US Government Printing Office, Washington, DC, USA, 1964.

[16] Á. Elbert, "Some recent results on the zeros of Bessel functions and orthogonal polynomials," Journal of Computational and Applied Mathematics, vol. 133, no. 1-2, pp. 65-83, 2001.

[17] R. Fletcher, Practical Methods of Optimization, Wiley, New York, NY, USA, 1987.

[18] O. Herrmann, L. R. Rabiner, and D. S. K. Chan, "Practical design rules for optimum finite impulse response low-pass digital filters," Bell System Technical Journal, vol. 52, no. 6, pp. 769-799, 1973.

[19] J. F. Kaiser and R. W. Schafer, "On the use of the $\mathrm{I}_{0}$-sinh window for spectrum analysis," IEEE Trans. Acoustics, Speech, and Signal Processing, vol. 28, no. 1, pp. 105-107, 1980.

[20] S. W. A. Bergen and A. Antoniou, "Nonrecursive digital filter design using the ultraspherical window," in Proc. IEEE Pacific Rim Conference on Communications, Computers and Signal Processing (PACRIM '03), vol. 1, pp. 260-263, Victoria, BC, Canada, August 2003.

[21] A. Antoniou, Digital Filters: Analysis, Design, and Applications, McGraw-Hill, New York, NY, USA, 1993.

[22] C. A. Balanis, Antenna Theory: Analysis and Design, Wiley, New York, NY, USA, 1982.

[23] G. G. Kuperman and T. D. Penrod, "Evaluation of compressed synthetic aperture radar imagery," in Proc. IEEE National Aerospace and Electronics Conference (NAECON '94), vol. 1, pp. 319-326, Dayton, Ohio, USA, May 1994.

[24] D. C. Munson, J. D. O’Brien, and W. K. Jenkins, "A tomographic formulation of spotlight-mode synthetic aperture radar," Proceedings of the IEEE, vol. 71, no. 8, pp. 917-925, 1983.

[25] H. Jiang, W. R. Chen, and H. Liu, "Techniques to improve the accuracy and to reduce the variance in noise power spectrum measurement," IEEE Trans. on Biomedical Engineering, vol. 49, no. 11, pp. 1270-1278, 2002.

[26] J. W. Adams, "A new optimal window," IEEE Trans. Signal Processing, vol. 39, no. 8, pp. 1753-1769, 1991.

Stuart W. A. Bergen was born in Guildford, England, UK, on November 5, 1976. He received the B.S. degree in electrical engineering from the University of Calgary, Calgary, Alberta, Canada, in 1999. Currently, he is pursuing the M.A.Sc. degree in electrical engineering at the University of Victoria, Victoria, British Columbia, Canada. From 1997 to 1998 , he was a firmware/hardware designer at Wireless Matrix, Calgary, Al-

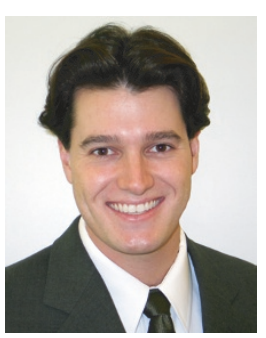
berta, Canada, focusing on satellite telecommunications for the oil and gas industry. From 1998 to 2000, he was a firmware design engineer at Nortel Networks, Calgary, Alberta, Canada, concentrating on digital signal processing (DSP) for telecommunications systems. His research interests include DSP algorithms, digital filter design, multirate signal processing, and beamforming for use in telecommunication, biomedical, and geophysics applications.
Andreas Antoniou received the B.S.(Eng.) and $\mathrm{Ph} . \mathrm{D}$. degrees in electrical engineering from the University of London in 1963 and 1966, respectively. He is a Fellow of the IEE and the IEEE. He taught at Concordia University from 1970 to 1983 , was the founding Chair of the Department of Electrical and Computer Engineering, University of Victoria, BC, Canada, from 1983 to 1990, and is now Professor Emeritus. His teach-

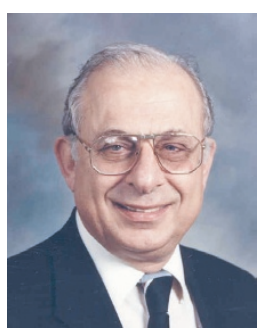
ing and research interests are in the area of digital signal processing. He is the author of Digital Filters: Analysis, Design, and Applications published by McGraw-Hill. Dr. Antoniou served as Associate/Chief Editor for IEEE Transactions on Circuits and Systems (CAS) from 1983 to 1987, as a Distinguished Lecturer of the IEEE Signal Processing Society in 2003, and as General Chair of the 2004 International Symposium on Circuits and Systems. He received the Ambrose Fleming Premium for 1964 from the IEE (Best Paper Award), a CAS Golden Jubilee Medal from the IEEE Circuits and Systems Society, the BC Science Council Chairman's Award for Career Achievement for 2000, and the Doctor Honoris Causa degree from the Metsovio National Technical University, Athens, Greece, in 2002 . 